\title{
Heartbeat: Beta-blockers for aortic regurgitation
}

The current paradigm for the pathophysiology of chronic severe aortic regurgitation (AR) is that chronic volume overload results in progressive left ventricular (LV) dilation with eventual symptom onset or asymptomatic LV systolic dysfunction. Clinical management focuses on periodic monitoring of LV size and ejection fraction, as well as symptoms, to ensure aortic valve replacement is performed just before the onset of irreversible LV contractile dysfunction. This paradigm suggests that medical therapy to reduce volume overload or block LV dilation would slow disease progression. Disappointingly, previous studies of medical therapy in adults with chronic severe AR have failed to show a significant effect on clinical outcomes.

In this issue of Heart, Broch and colleagues (see page 191) hypothesized that beta-blocker therapy would reverse LV dilation in patients with chronic AR, similar to the effects of beta-blockers in patients with heart failure with reduced ejection fraction. To test this hypothesis, they performed a double blind clinical trial of metoprolol versus placebo in 75 asymptomatic adults (mean age 44 years, $89 \%$ male) with chronic moderate-severe AR using both echocardiographic and cardiac magnetic resonance (CMR) imaging measures of AR severity and LV geometry. Although, there were no serious adverse effects of metoprolol therapy, after 6 months there was no difference between groups in LV enddiastolic volume, exercise capacity or peak oxygen consumption. The only significant differences were a 2.7 (95\% CI 0.1 to 5.3 ) percentage points higher LV ejection fraction and 138 (95\% CI 71 to 205) $\mathrm{pg} / \mathrm{mL}$ higher $(\mathrm{p}<0.001) \mathrm{N}$-terminal pro-B-type natriuretic peptide serum level in those randomized to metoprolol compared to the placebo group (figure 1).

The effects of beta-blockade in chronic $\mathrm{AR}$ are complicated by changes in valve hemodynamics at a slower heart rate. With a longer diastolic time interval, there is a larger regurgitant volume per beat, although probably no change in overall regurgitation when averaged over many beats. In theory, the total LV stroke volume ejected across the aortic valve on each beat should increase to maintain a

\section{Catherine M Otto}

normal forward cardiac output, resulting in a wider pulse pressure. However, in this study, the lower resting heart rate with metoprolol therapy $(55 \pm 17$ vs 62 $\pm 18 \mathrm{bpm}, \mathrm{p}=0.02)$ was accompanied by a lower systolic $(124 \pm 17$ vs $134 \pm 19 \mathrm{~mm}$ $\mathrm{Hg}, \mathrm{p}=0.01)$ and lower diastolic $(58 \pm 9$ vs $67 \pm 6 \mathrm{bpm}, \mathrm{p}<0.001)$ blood pressure at rest, with similar pulse pressures in both groups. These findings suggest that historical concerns about hemodynamic effects of beta-blockers in chronic AR may not be justified.

Do the negative results of this study mean that beta blocker therapy for chronic AR is not beneficial or does the absence of adverse effects suggest we need larger, longer term studies with clinical endpoints? In an accompanying editorial (see page 168), Evangelista reviews experimental and clinical data supporting the potential benefit of beta-blockade in chronic AR, noting that previous epidemiological studies are difficult to interpret given the high prevalence of concurrent cardiac and noncardiac conditions in these populations. He points out that LV dilation in chronic AR occurs very slowly over a long time period so that the short follow-up and small sample size of
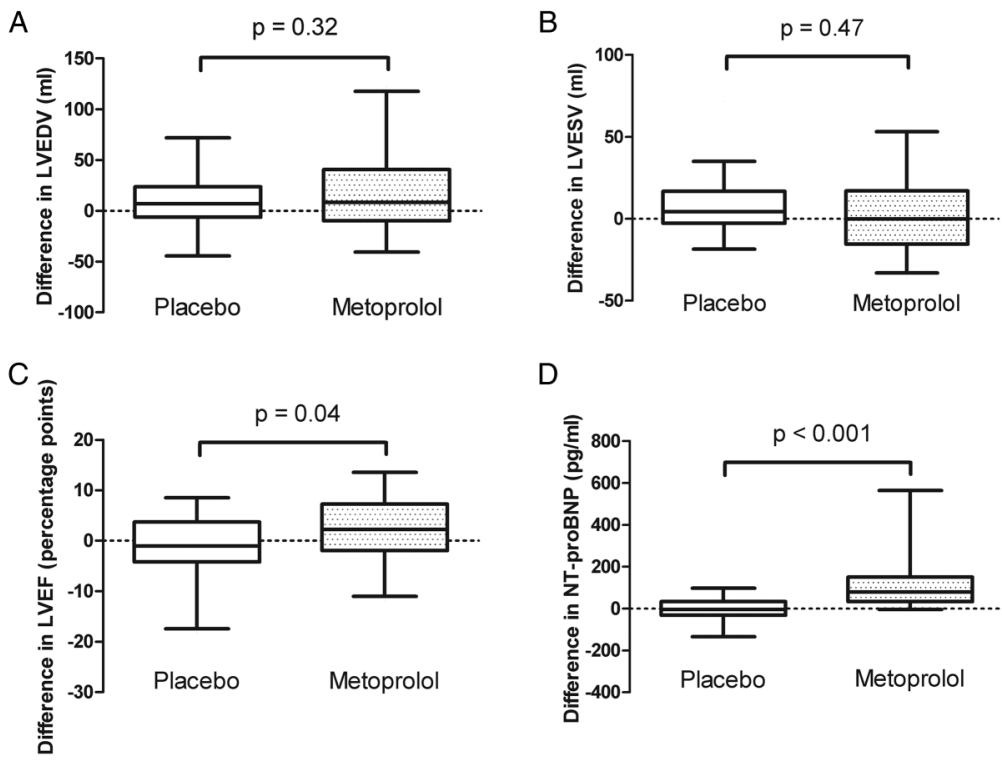

D

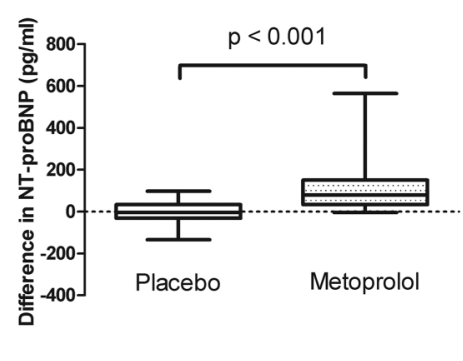

Figure 1 Changes in main outcome variables from baseline to follow-up. Panels show the mean (whiskers 5-95\% interval) changes in (A) left ventricular end diastolic volume (LVEDV), (B) left ventricular end systolic volume (LVESV), (C) left ventricular ejection fraction (LVEF), and (D) N-terminal pro-B-type natriuretic peptide (NT-proBNP) in patients allocated to metoprolol and placebo.

the current study may have been inadequate to detect a significant effect of beta blocker therapy. He concludes that "a large trial with a long period of treatment is required to ascertain the benefit of betablocker treatment on the reduction or delay in surgical aortic valve treatment indication, in patients with asymptomatic severe aortic regurgitation”. Further, I suggest that the simplistic paradigm that LV dilation in chronic AR is driven only by chronic volume overload is incorrect. We need to consider that disease progression in chronic AR, like other forms of heart failure, is due to a complex interplay between valve hemodynamics, the LV hormonal changes.

Reduction of serum low density lipoprotein cholesterol (LDL-C) is central to medical therapy for primary and secondary prevention of cardiovascular disease. Niacin is a potent medication that lowers both LDL-C and triglycerides as well as increasing high density lipoprotein cholesterol (HDL-C) levels. Despite these desirable effects on serum lipid levels, niacin therapy has several disadvantages, particular in comparison with other cholesterol lowering medication. One area of concern 


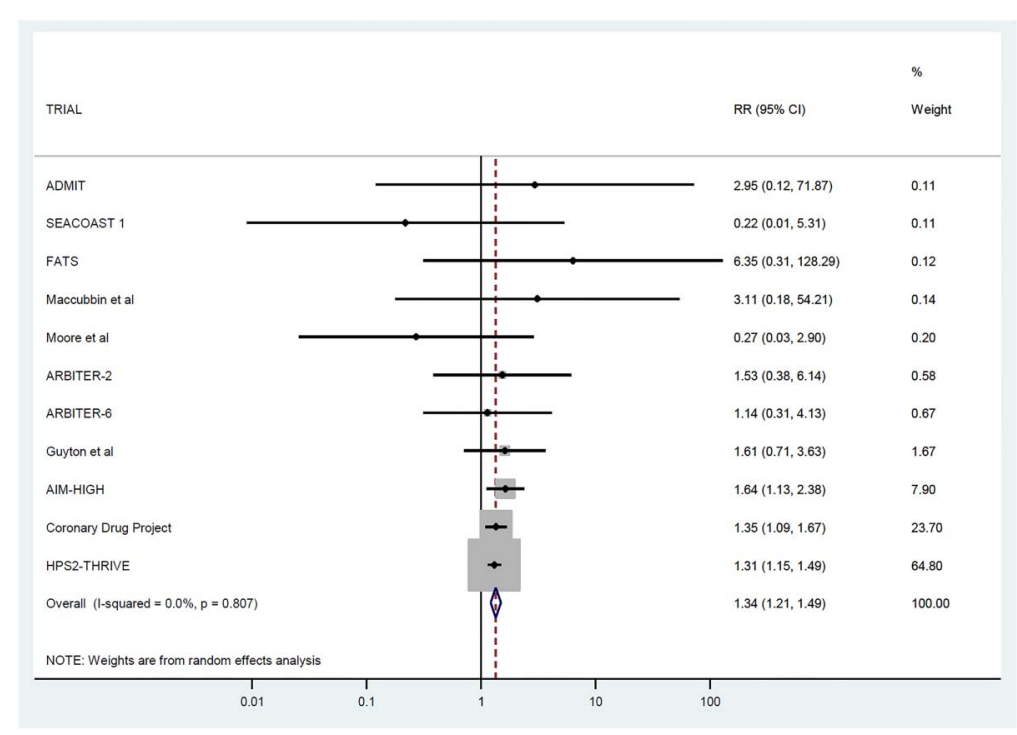

Figure 2 Association between niacin therapy and incident diabetes in 11 trials. (Ordered by weight contributed to meta-analysis.) AIM-HIGH, Atherothrombosis Intervention in Metabolic Syndrome with Low HDL/High Triglycerides: Impact on Global Health Outcomes; HPS2-THRIVE, Heart Protection Study 2-Treatment of HDL to Reduce the Incidence of Vascular Events.

is the association of niacin therapy with an increased risk of new onset diabetes. In this issue of Heart, a meta-analysis of 11 randomized controlled clinical trials including over 26 thousand patients (see page 198) found that niacin treatment resulted in one additional case of diabetes per 43 (95\% CI 30 to 70) initially nondiabetic patients, corresponding to a relative risk of 1.34 (95\% CIs 1.21 to 1.49 ) for new-onset diabetes with niacin therapy (figure 2). These findings were not affected by background statin therapy or combined therapy with laropiprant (which is taken to reduce niacin-induced flushing).

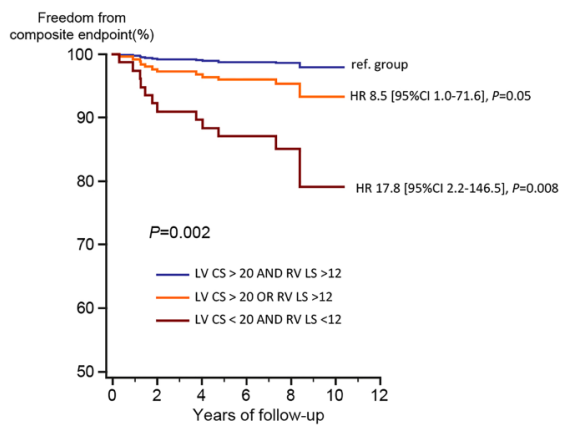

Figure 3 Cox-analysis based survivor function for the freedom of cardiac death, successful resuscitation or documented ventricular tachycardia (=pre-defined combined endpoint) based on left ventricular circumferential and right ventricular longitudinal strain. $\mathrm{CS}=$ circumferential strain on feature tracking cardiac magnetic resonance imaging, $L V=l e f t$ ventricle.
In an editorial, Haynes and Rahimi (see page 170), note that the risk of new onset diabetes is only about $10 \%$ with statin therapy compared to to the $30 \%$ increased risk with niacin therapy as documented in this meta-analysis. In addition, niacin is not well tolerated with over $30 \%$ of patients being unable to take full dose therapy due to intolerable side effects such as flushing, pruritus and gastrointestinal symptoms. Niacin therapy also is associated with an increased risk of infection and bleeding. Further, recent studies have not shown a significant reduction in cardiovascular risk with the addition of niacin to standard medical therapy.

As Haynes and Rahimi so elegantly summarize: "The decision whether to use any treatment always involves a balance of benefits and risks. The data now available for niacin suggest that there does not appear to be any significant cardiovascular benefit when niacin is used in statintreated patients, but there are very real hazards. Disappointing as this is for patients and physicians (who both want additional treatments that can improve outcomes), it again highlights the fallacy of assuming that treatments with encouraging effects on surrogate outcomes (such as HDL-C or other components of the lipid profile) will be of net clinical benefit to patients in terms of hard clinical outcomes. However, such disappointment does not justify continuing use of a treatment with no obvious clinical benefit and real harm. Physicians who have used niacin to assist in the achievement of guideline-directed lipid "goals" must recognise that practice needs to change in the light of evidence. This is not to say that there is absolutely no role for niacin in modern treatment, but any role remains to be proven and cannot be assumed. Regulators need to reconsider previous decisions about niacin and guidelines must be updated, but the onus remains on individual physicians: primum non nocere
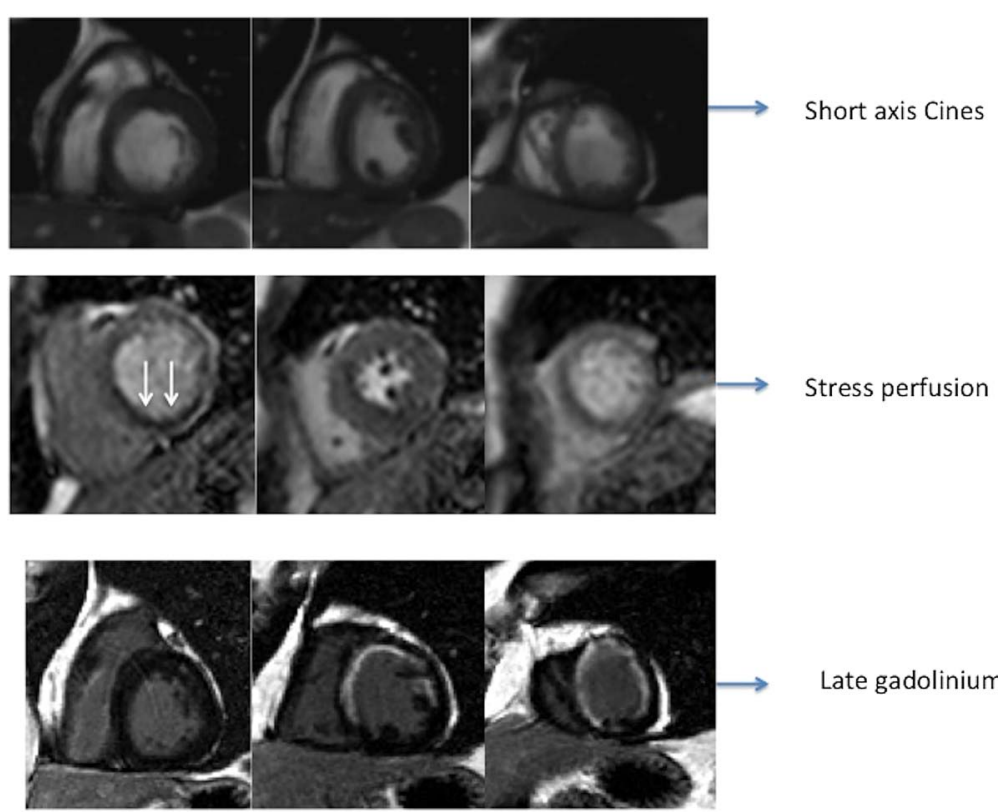

Late gadolinium

Figure 4 Assessment of chronic ischaemic heart disease: A patient with previous anterior myocardial infarction assessed for ischaemic heart disease. Stress perfusion imaging showed basal inferior perfusion defect (arrow), and late enhancement imaging showed transmural myocardial infarction in the mid-distal LAD territory with viable inferior wall. 
(first do no harm). In the case of niacin, it would appear this would translate into avoiding its use unless there are very good reasons not to."

Advanced cardiac imaging can provide a variety of sophisticated measures of cardiac function but data often is lacking on clinical utility of these parameters. Feature tracking cardiac magnetic resonance (CMR) imaging now allows direct quantitation of myocardial deformation with measurement of global longitudinal (LS), circumferential (CS) and radial strain (RS) for both the left (LV) and right ventricle (RV). In a series of 372 tetralogy of Fallot patients (mean age 18 years) over 7.4 years of follow-up, 20 (5.4\%) experienced a combined endpoint of death, successful resuscitation or documented ventricular tachycardia (see page 209). Independent predictors of this combined clinical endpoint were LV CS and RV LS, with this association being independent of QRS duration, LV and RV ejection fractions and volumes, NYHA class and peak oxygen uptake. The combination of an LV $\mathrm{CS} \leq 20$ and and RV $\mathrm{LS} \leq 12$ identified a subgroup of tetralogy patients with a greater than 3 -fold increased risk of the combined endpoint, compared to the remainder of group (figure 3). As this study demonstrates, validation of imaging parameters as predictors of clinical outcomes provides much stronger evidence for clinical decision making than simply comparing one imaging modality to another.

The Education in Heart article in this issue (see page 239) provides a primer on the use of CMR imaging for assessment of ischemic heart disease. In patients with coronary disease, CMR is particularly useful for assessment for myocardial edema, fibrosis and perfusion, as well as for accurate measurement of LV volumes and ejection fraction. CMR imaging with dobutamine stress can be used for detection of myocardial viability (at low dose) and inducible ischemia (at higher doses) (figure 4).

Check out the Image Challenge case; it is unusual but not too difficult, Once you have seen it you will make the correct diagnosis in your clinical practice should you encounter this diagnosis yourself in the future (see page 238).

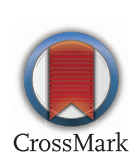

To cite Otto CM. Heart 2016;102:165-167.

Heart 2016;102:165-167.

doi:10.1136/heartjnl-2015-309186 